\title{
Flexible Embedded Telemetry System for Agriculture and Aquaculture
}

\author{
André Weiskopf ${ }^{1}$, Frank Weichert ${ }^{1}$, Norbert Fränzel ${ }^{2}$, Manuel Schneider $^{3}$ \\ ${ }^{1}$ Fraunhofer Advanced System Technology (AST), \\ Am Vogelherd 50, 98693 Ilmenau, Germany \\ \{andre.weiskopf, frank. weichert\} @iosb-ast. fraunhofer.de \\ ${ }^{2}$ Technical University Ilmenau, Ehrenbergstraße 29, 98693 Ilmenau, Germany \\ norbert.fraenzelatu-ilmenau.de \\ ${ }^{3}$ University of Applied Science Schmalkalden, \\ Blechhammer 9, 98674 Schmalkalden, Germany \\ m.schneider@fh-sm.de
}

\begin{abstract}
This paper describes a system for data acquisition and remote maintenance via wireless communication. In this work the concept of managing time critical tasks via networks is shown. The system consists of a client-serverstructure with a microcontroller-based module connected to the user devices and maintenance software, both as clients and a maintenance server for establishing the connection between the clients and to identify users. The special interest in this paper is the implementation of a specialized communication protocol to optimize the communication between the clients.
\end{abstract}

Keywords: Telemetry, ISOBUS, CAN, remote maintenance, wireless communication, real-time, tracking, time-critical process.

\section{$1 \quad$ Introduction}

The options for diagnosis and maintenance by software have raised rapidly with the growing number of embedded systems in all kinds of products. By changing the parameterization or the firmware of embedded systems a lot of adaptions are possible. These tasks can be done for stationary systems and by an increasing availability of wireless communication services also remotely for mobile systems. The use of remote maintenance services reduces downtimes and travel expenses for service personnel.

In this paper a system is described for both the diagnosis and remote maintenance of stationary systems, e.g. greenhouses and fish farming, and additionally fleet management of mobile systems, e.g. agriculture machines like harvester. In modern automation and automotive technology a lot of different digital systems are used e.g. for control of systems or parts thereof. These consist of individual modules which communicate via a common data bus, i.e. CAN, ISOBUS, Ethernet or RS485. To fulfill the different tasks various combinations of input-, output, sensor- and actor modules are possible. By an increasing number of software parameters in these modules the overall systems are becoming increasingly complex. For an optimal 
adaption to the requirements of the task the modules can be parameterized in large scale. At the same time the modules can be used for reading sensor values to make this information available to other components of the system or to the service personnel. By using the internal error history and monitoring feature of the different modules troubleshooting is supported. The diagnosis and maintenance of such systems demand comprehensive knowledge by specially trained personnel to fulfill the tasks.

The technology "FETS" can be used for remote maintenance and diagnosis of such control systems. "FETS" stands for Flexible Embedded Telemetry System. It can be adapted to various stationary and also mobile systems. The monitoring of the individual systems can take place via a service control center. The development of a command pattern, which allows control of time critical processes on the CAN bus side of a system over communication networks without Quality of Service (QoS), was one main challenge to solve. With this technology the service personnel has the opportunity to upload new firmware to the modules, which have to be remotely maintained. In this way new features can easily be implemented and the behavior can be adjusted, so the range of functions increases, e.g. by using new sensor or actor modules.

In modern agriculture the ISOBUS is of growing importance [1, 2, 3]. ISOBUS is defined by $250 \mathrm{kbaud}$ CAN-2.0b communication protocol based on the ISO11783 norm $[4,5,6]$. At this various auxiliary equipment will be connected to the agriculture machine, e.g. modern tractor or harvester, via a common data bus. In this way information like driving speed, hydraulic pressures and valve positions are made available by the Electronic Control Unit (ECU) of the agriculture machine. Depending on the number and types of the auxiliary equipment the agriculture will be more efficient by resource-conserving use of plant protection products. In a further expansion phase of the system the remote control of agriculture machines with certain limits is possible. This will be examined at precision farming. In addition to using FETS for mobile systems in agriculture the technology can be applied to stationary systems, i.e. control of greenhouses and aquaculture by monitoring of vital factors like oxygen concentration, $\mathrm{pH}$ value and temperature.

Currently the technology FETS is applied as remote maintenance system for modern electrical wheelchairs, as tracking system for pedelec hire and also as maintenance and diagnosis system for solar power plants. For adaption to various user interfaces FETS is based on a modular design principle.

\section{Related Work}

Mainly in the automotive sector the use of mobile communication networks for maintenance tasks is discussed, e.g. [7]. In [8] an idea to connect a local onboard module to a remote server is presented. In the field of maintenance and monitoring for a variety of purposes numerous reports exist, e.g. in $[9,10]$ mobile communication is used for monitoring a wheelchair and in [11] for automated alarming if a failure is detected. Bidirectional communication is discussed in [12] in the area of additional services. 
With regards to the focus of this paper, the remote maintenance of agriculture machines and monitoring of greenhouse and aquaculture, a few reports exists. In [1, 2, 3] some aspects for embedded systems, which are used for communication with agricultural machines via ISOBUS, are given.

\section{System Overview}

The developed technology is based on three main components. Figure 1 shows a simplified overview of the system.

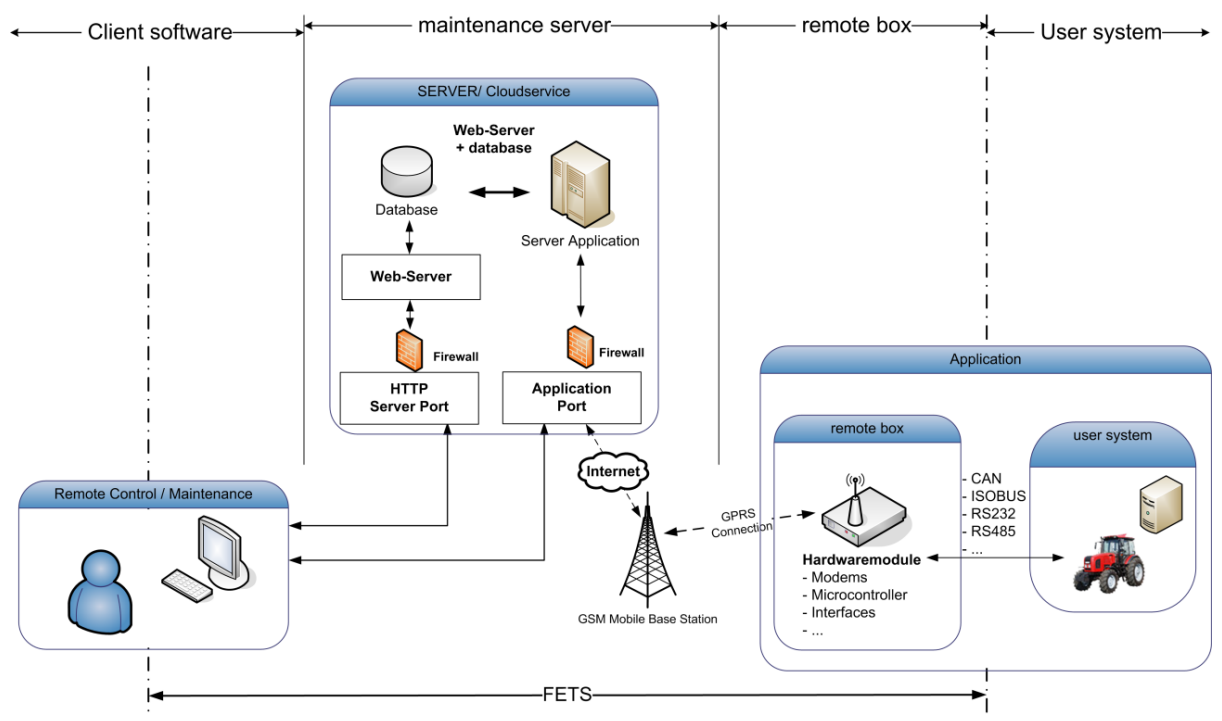

Fig. 1. Overall system overview

The first component, called "remote-box", is a typical embedded system based on a Microcontroller which offers sufficient communication interfaces and hardware resources to fulfill all tasks of remote maintenance. This box realizes the time critical communication with the data bus of the system that has to be maintained. Another key aspect is the opportunity to connect to a server via GPRS-based communication. Within the "FETS" technology modular software was designed. With an individual adaption of the corresponding hardware the firmware can be ported easily onto other Microcontrollers. The expansion phase of the remote box depends on the boundary conditions of the user system. This contains for instance the time behavior of the system, the number of components to be monitored and configured as well as the required calculation time. In addition the box may contain a GPS receiver, so the localization of mobile systems is possible. Furthermore it could be equipped with RFcomponents, so the box can be used as a network node or transmit data via ISM-Band. In this case the transfer of data can be encrypted by using crypto-algorithms. Figure 2 illustrates a used expansion stage of the hardware of the remote-box. 


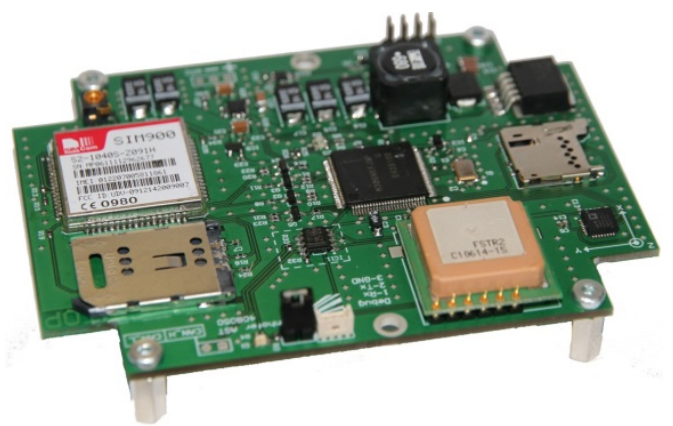

Fig. 2. Hardware of the remote-box

The server is the central communication relay between the clients (remote-boxes and maintenance software). The server application verifies the authorization status of the actual user. So it can decide which user has access to which remote box. After that the user can connect to a selected remote-box. Now the server establishes a transparent connection between remote-box and maintenance client software. It is important to note that the whole data is transmitted via the server and not point-topoint. So, only the server needs a static, visible internet address. Additionally the server will be used for databases and updates. So the remote-boxes and client-PCs can download the most up to date software. By integrating automatic updates the firmware of the remote-boxes and software of the maintenance client-PCs are always up-to-date. This ensures that no different versions of software will be used, so the system maintenance gets more efficient.

\section{Research Issue}

The main task is to connect the time-critical communication of the end devices with the latency afflicted communication via GPRS to the server. In picture 3 a histogram of the transfer of data packets with typical latencies of GPRS communication is shown.

The measurements in the histogram are for the transmission of a single data packet from remote-box to server and back. So an average transfer of a single packet is around 750 milliseconds, but it is also possible that this data transfer time is much higher.

In CAN-Bus based systems the data exchange between different nodes is organized by service data objects (SDO) and process data objects (PDO). For example by connecting the remote-box to a CAN-BUS based system, typical timeouts for answering to service data objects are 250 milliseconds. To process time critical commands on the CAN-Bus it is necessary to know exactly the maximal timeouts. If such a timeout is exceeded, it is also possible that warnings, errors in or shut-offs of the user system can be produced. 


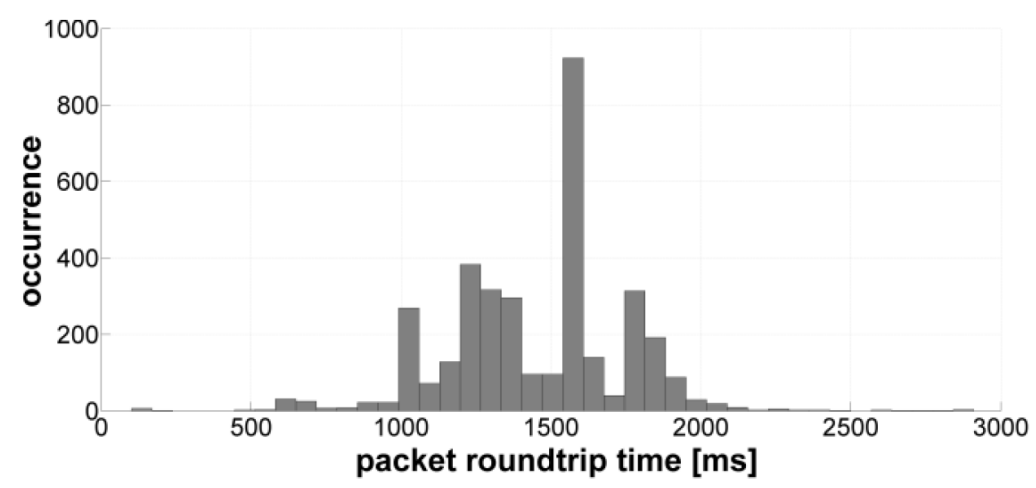

Fig. 3. Histogram of packet transfer via GPRS

Due to different latencies of transfers via GPRS and CAN, a direct transfer from the server to the remote-box is not reasonable.

\section{$5 \quad$ Design of the Communication Process}

To solve the problem with the different latencies a specialized macro language was included. With these macros it is possible to execute time critical operations selfcontained. Therefore a specialized communication protocol stack is integrated in all 3 parts of the remote maintenance system. Its job is to allocate different services for time-critical processes.

This stack is based on the TCP-Stack. To optimize the communication process a packet manager and action manager are added to the TCP-Stack. Figure 4 shows the schematic of the communication process.

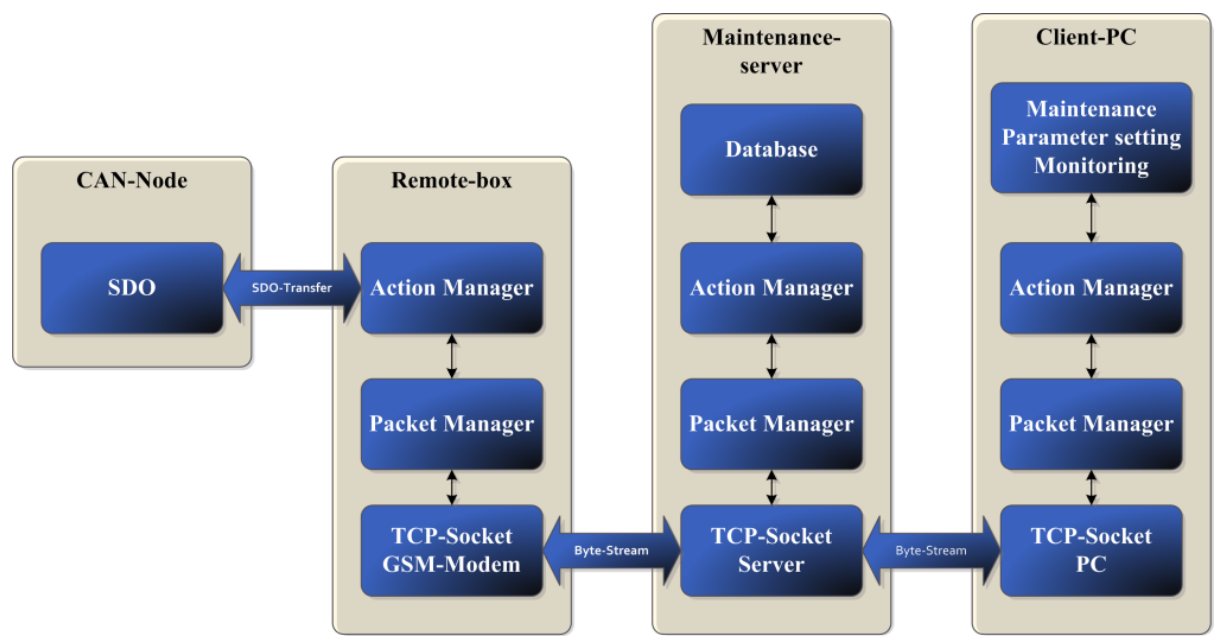

Fig. 4. Structure of communication process 
The action manager layer executes actions as commands and macros. That means its job is the data processing in PC, server and remote-box. On the remote-box side typical actions are reading out complete list of parameters, measurements or setting new parameters and on PC-side to display measurements or to indicate errors. The most critical part is to keep the user system in a secure state at all time. Therefore it is necessary to transfer all data from the maintenance software to remote-box first and after that to process the data in an appropriate manner. The TCP socket is included in the PC's and also in the firmware of embedded modems. Its job is to transfer the data between the 3 system parts. TCP by itself provides a secure byte-stream, but during the process of design there are some restrictions like the loss of the connection in wireless systems or another typical problem is the overflow of buffers for the received data in the modem.

To optimize the time of complete data transfer a packet manager is included between TCP socket and action manager. Its job is to divide the complete data stream of the transmitter in single data packets, to include some control bytes in these packets and also to summarize the received single data packets to one data stream.

A whole data packet consists of an info field, an ID-field, the macro command, and the data to be transmitted. The packet manager is also responsible for detecting lost packets. So it can react to this by different actions like packet repeats.

Most processes in user systems are designed to use simple commands. So it is usually enough to send one data packet like readout one measurement or set one parameter. The structure of this process is shown in figure 5a). Some processes require more communication, e.g. firmware updates or the transmission of a whole parameter set. In this case typically two transfer mechanisms are used. The first is to send a confirmation after each packet and send then the second data packet and so on, see figure $5 \mathrm{~b}$ ). The second one is to send the whole byte-stream without confirmation. Only one confirmation is send when the data stream is finished, see $5 \mathrm{c}$ ). Here it is possible to loose single packets and thus it is necessary to send the whole data stream again.

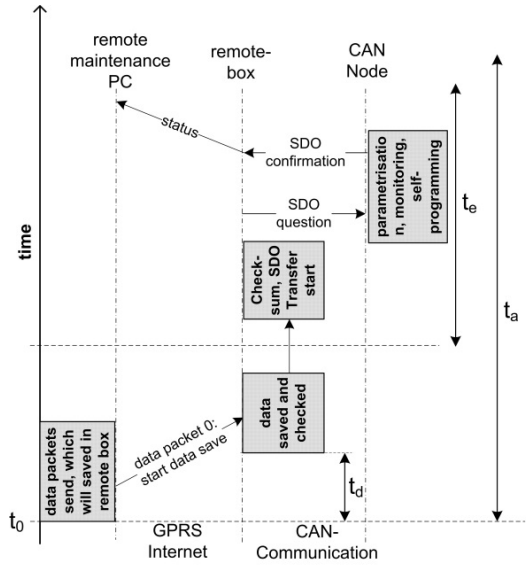

a)

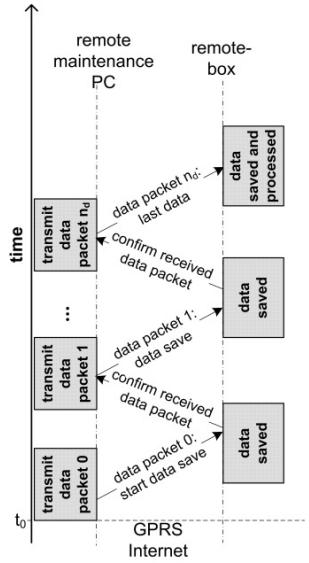

b)

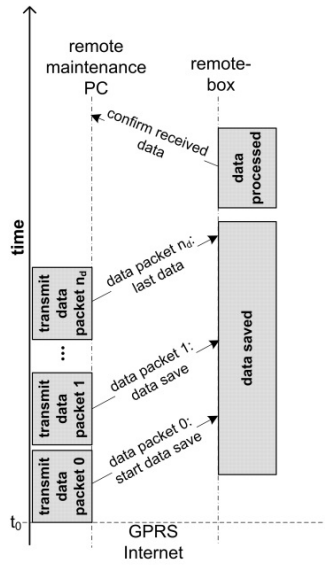

c)

Fig. 5. Standard transmit processes 
In the FETS Technology a mixture of both processes is used to optimize the transfer. In the remote maintenance system all the data packets are send in series. And for every single data packet a confirmation with the ID is transmitted backwards. So it is possible to identify lost packets. With this principle it is not necessary to repeat the whole byte-stream again. The only packets to repeat are these packets where no confirmation was transmitted backwards. In figure 6 this new approach is shown.

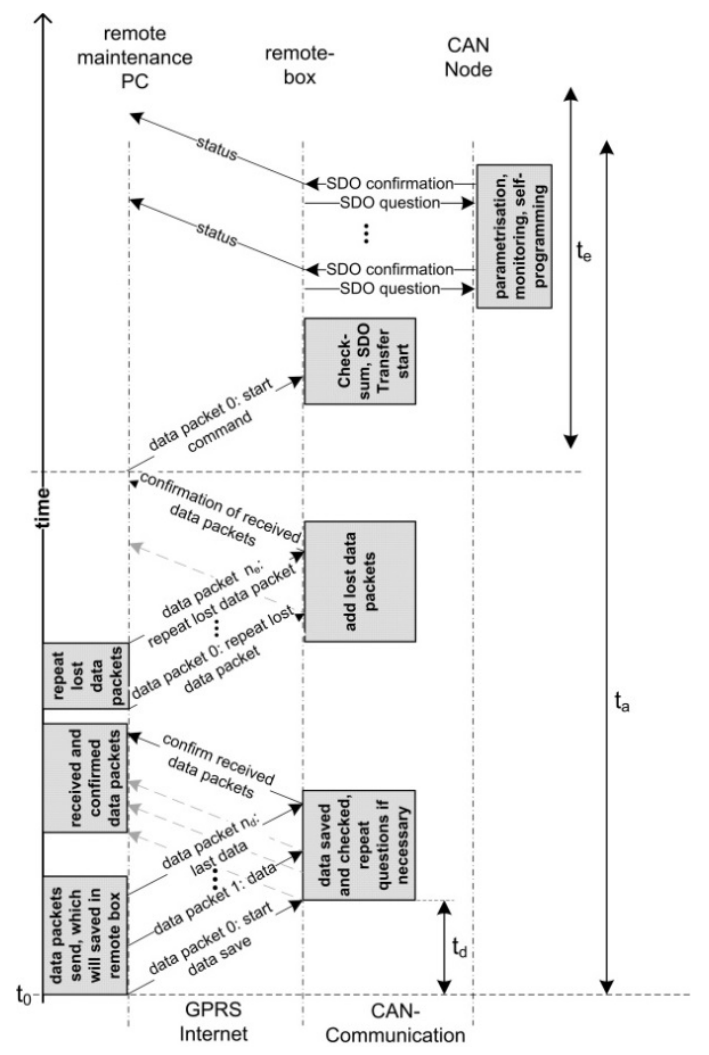

Fig. 6. Transmit process at FETS technology

This concept is especially beneficial if big amount of data has to be transferred. Big means in this context for example a complete firmware update where the SDOprocess on the CAN-Bus is perhaps 10.000 messages long. The same problem exists with the transfer of a complete set of parameters to the user system or reading out all measurements of the user system at once. In the case of small byte-streams there is a little overhead with this concept, but it is insignificant compared to the payload.

\section{Conclusion and Acknowledgements}

A fast, easy and safe transmission of data via GPRS cannot be guaranteed. To prevent the accumulation of long waiting periods the communication dialog should be 
reduced to realize an efficient communication with user system via remote maintenance technology.

Here, the maintenance system was extended by a special protocol stack. This was realized on the one side in the firmware for the remote-box and on the other side in the application software for the client PC. The task is to summarize the time-critical command sequences (actions) in packets in the remote-box. So a secure time critical communication with the system can be ensured.

With this technology a Client-Server-Structure was realized, where the remoteboxes and Maintenance-PCs communicate via a specific server as clients. A specifically developed application is the basis of the server. This accepts incoming connection requests from the clients, manages the user authentication and transfers data packets between the clients which are connected. Specific data packets can be saved in a database. Although the connection between Maintenance-PC and remote box is established through a server, there is a transparent connection between them. The server is the central exchange.

A special software design is needed for the resulting complexity of the firmware with many parallel processes. A special multitasking system was set up to obtain functional embedded software with the used hardware. This design pattern allows an efficient use of the processor resources with the help of nested interrupts.

The developed technology "FETS" is an approach for the realization of maintenance and remote diagnostic system. By the presented concept a system was developed, which can easily be adapted to a variety of different user systems. The technology is not limited to CAN based systems, it can easily be adapted to other interfaces and communication protocols.

FETS has already been successfully tested in a field test phase in Europe as a localization-, diagnosis- and maintenance module on electrical wheelchairs and pedelecs. A present field of application is the maintenance of solar power plants. It also became obvious, that the technology is very robust and reliable. So the application of this technology is interesting for the maintenance and diagnosis of vehicles and stationary systems.

\section{References}

1. Fantuzzi, C., Marzani, S., Secchi, C., Ruggeri, M.: A Distributed Embedded Control System for Agricultural Machines. In: IEEE International Conference on Industrial Informatics, Singapore (2006)

2. Zhang, M., Zhou, Z., Xi, Z.: In: Second International Conference on Intelligent Computation Technology and Automation (ICICTA), Cangsha (2009)

3. Sarker, M.K., Park, D.S., Badarch, L.: Electronic Control Sensors Applications for the Next Generation Tractor Based on Open Source Library. In: Sixth Conference on Sensing Technology (ICST), Kolkata (2012)

4. Tractors and machinery for agriculture and forestry', International Standard ISO 11783-1 to 13 , ISO (2007)

5. Felimeth, P.: CAN-based tractor- agricultural implement communication ISO 11783, CAN-Newsletter 9 (2003) 
6. Stone, M.L.: ISO 11783 - An Electronic Communications Protocol for Agricultural Equipment. ASEA Distinguished Lecture Series (1999)

7. Herrtwich, R.G.: Automotive Telematics - Road Safety versus IT Security? In: International Conference on Computer Safety, Reliability and Security, Potsdam (2004)

8. Zhang, Y., Salman, M., Subramania, H.S., Edwards, R., Correia, J., Gantt, G.W., Rychlinski, M., Stanford, J.: Remote Vehicle State of Health Monitoring and Its Application to Vehicle No-Start Prediction. In: IEEE Autotestcon, Anaheim (2009)

9. Touati, Y., Ali-Cherif, A., Achili, B.: Smart Wheelchair Design and Monitoring via Wired and Wireless Networks. In: IEEE Symposium on Industrial Electronics and Applications, Kuala Lumpur (2009)

10. Sevillano, J.L., Cascado, D., Vicente, S., Lujan, C.D., del Rio, F.D.: A Real-Time Wireless Sensor Network for Wheelchair Navigation. In: International Conference on Computer Systems and Applications, Rabat (2009)

11. Lobardi, P., Giaconia, C.G., Di Dio, V.: An Embedded Diagnostic System for Wheelchairs Brushless Drives Monitoring. In: International Symposium on Power Electronics, Electrical Drives, Automation and Motion, Taormina (2006)

12. Zhihong, T., Jinsheng, Y., Jianguo, Z.: Location Based Services Applied to an Electrical Wheelchair Based on the GPS and GSM Networks. In: International Workshop on Intelligent Systems and Applications, Wuhan (2009) 